\title{
DOI: 10.33947/1982-3282-v15n1-2-4366 \\ DIABETES GESTACIONAL EM GRAVIDEZ GEMELAR: RELATO DE CASO
}

\author{
GESTATIONAL DIABETES IN TWIN PREGNAC: CASE REPORT
}

\section{DIABETES GESTACIONALES EN EMBARAZO GEMELAR: REPORTE DE UN CASO}

\author{
Bárbara Kellen Antunes Borges', Franscisleidy Queiroz dos Santos², \\ Selma Cecília Gonçalves de Souza ${ }^{3}$, Thiago Alves Xavier dos Santos ${ }^{4}$
}

Submetido: $19 / 05 / 2020$

Aprovado: 12/12/2020

\section{RESUMO}

Introdução: O Diabetes Mellitus Gestacional (DMG) refere-se a qualquer intolerância à glicose, de importância variável, com início ou diagnóstico durante a gestação. A gravidez gemelar é uma considerada de risco para o feto e para a mãe por causar prematuridade neonatal, baixo peso ao nascer, malformações fetais, anemia materna e pré-eclâmpsia. Objetivo: Objetivou-se relatar o caso de uma gestante portadora de DMG em gravidez gemelar. Resultados: Estudo de caso único, com paciente de 33 anos, com gravidez gemelar, portadora de DMG, que antes de ser diagnosticada não se preocupava com a alimentação e após o diagnóstico da DMG alterou sua rotina alimentar. Conclusão: Verificou-se que a paciente tinha entendimento sobre a DMG e isso influenciou em uma mudança de seus hábitos alimentares na busca por uma gestação com menos riscos maternos e neonatais e qualidade de vida.

DESCRITORES: Diabetes Gestacional, Gravidez de Gêmeos, Fatores de risco, Relato de Caso.

\begin{abstract}
Introduction: Gestational Diabetes Mellitus (GDM) refers to any glucose intolerance, of varying importance, with onset or diagnosis during pregnancy. Twin pregnancies are considered risky for the fetus and the mother because they cause neonatal prematurity, low birth weight, fetal malformations, maternal anemia and pre-eclampsia. Objective: The objective was to report the case of a pregnant woman with GDM in twin pregnancies. Results: $A$ single case study with a 33-year-old patient with twin pregnancies, with GDM, whom before being diagnosed did not care about food and after the GDM diagnosis changed her eating routine. Conclusion: It was found that the patient had an understanding of GDM and this influenced a change in her eating habits in the search for a pregnancy with less maternal and neonatal risks and quality of life.
\end{abstract}

DESCRIPTORS: Gestational Diabetes, Twin Pregnancy, Risk Factors, Case Report.

\section{RESUMEN}

Introducción: la diabetes mellitus gestacional (DMG) se refiere a cualquier intolerancia a la glucosa, de importancia variable, con inicio o diagnóstico durante el embarazo. Los embarazos gemelares se consideran riesgosos para el feto y la madre porque causan prematuridad neonatal, bajo peso al nacer, malformaciones fetales, anemia materna y preeclampsia. Objetivo: El objetivo fue informar el caso de una mujer embarazada con DMG en embarazos ge-

\footnotetext{
${ }^{1}$ Doutora em Ciências Animal pela DMVP/UFMG. Docente das Faculdades de Saúde Ibituruna - FASI e Faculdades Unidas do Norte de Minas - FUNORTE. Professora Orientadora da Pós-graduação do Instituto Federal do Norte de Minas Gerais - IFNMG.

2 Discente do Curso de Biomedicina na Faculdade de Saúde Ibituruna - FASI, Montes Claros, Minas Gerais, Brasil.

${ }^{3}$ Discente do Curso de Biomedicina na Faculdade de Saúde Ibituruna - FASI, Montes Claros, Minas Gerais, Brasil.

${ }^{4}$ Farmacêutico, Docente no Instituto Educacional Santa Cruz - IESC, Montes Claros, Minas Gerais, Brasil.
} 
melares. Resultados: un estudio de caso único con una paciente de 33 años con embarazos gemelares, con DMG, que antes de ser diagnosticada no se preocupaba por la comida y después del diagnóstico de DMG cambió su rutina de alimentación. Conclusión: se encontró que la paciente tenía una comprensión de la DMG y esto influyó en un cambio en sus hábitos alimenticios en la búsqueda de un embarazo con menos riesgos maternos y neonatales y calidad de vida.

DESCRIPTORES: Diabetes gestacional, embarazo gemelar, factores de riesgo, reporte de caso.

\section{INTRODUÇÃO}

Diabetes Mellitus (DM) é uma definida pela elevação da glicose no sangue (hiperglicemia). Pode acontecer devido a problemas na secreção ou na ação do hormônio insulina, sendo a insulina responsável por permitir a entrada de glicose para as células do organismo de forma que ela possa ser utilizada para as diversas atividades celulares ${ }^{1}$.

A DM do tipo 1 (DM1) ocorre pela deficiência na secreção da insulina e acomete na maior parte as crianças e tem como maior causador os fatores genéticos ${ }^{2}$. Em um levantamento feito por Garcia et al. ${ }^{3}$, aproximadamente 90\% dos diagnósticos da DM1 são em crianças menores de 15 anos e isso pode resultar no aumento de risco de complicações precoce, levando a diminuição da expectativa de vida da criança.

Há a DM do tipo 2 (DM2) a mais comum e com crescente ascensão no mundo. É uma doença crônica caracterizada pela destruição autoimune das células $\beta$ do pâncreas ou por redução de sensibilidade da insulina ${ }^{2,3,4}$. Segundo o manual do Ministério da Saúde ${ }^{5}$, aproximadamente $90 \%$ dos pacientes adultos diagnosticados com DM no Brasil é a DM2, ainda para a International Diabetes Federation ${ }^{6}$ estima que no Brasil há cerca de 14,3 milhões de adultos com diabetes e no mundo em 2035 haverá 592 milhões de pessoas acometidas pela DM2. Os principais fatores de risco relacionados a DM2 são a Hipertensão Arterial Sistêmica (HAS), sedentarismo, aumento de peso e colesterol, histórico familiar, doenças renais crônicas, uso de medicamentos glicocorticoides ${ }^{4,5}$.

O manejo da DM2 é considerado difícil e requer uma educação contínua do paciente para manter o monitoramento constante dos níveis glicêmico, além da adesão e do acompanhamento ao tratamento medicamentoso juntamente com a realização atividades físicas e mudanças na dieta ${ }^{2,6,7,8}$.

O Diabetes Mellitus Gestacional (DMG) se refere a qualquer intolerância à glicose, de importância variável, com início ou durante a gestação. Similar ao DM2, o DMG associa-se tanto destruição autoimune das células $\beta$ do pâncreas ou por redução de sensibilidade da insulina. O DMG ocorre em 1 a $14 \%$ de todas as gestações e relaciona-se com aumento de morbidade e mortalidade perinatais no Brasil ${ }^{1,2}$.

A gestação gemelar é preocupante, principalmente para saúde pública, pois é considerada de risco para o feto e mãe. A literatura orienta que pode resultar em prematuridade neonatal, baixo peso ao nascer, malformações fetais e também risco de mortalidade e morbidade materna e do bebê são encontrados. Os gêmeos mostram riscos oito vezes maiores de parto abaixo de 32 semanas, três vezes maiores para restrição de crescimento fetal (RCF) e três vezes maior para mortalidade neonatal ${ }^{1,9,10}$. O presente estudo, objetivou relatar o caso de uma gestante portadora de DMG em gravidez gemelar.

\section{RELATO DE CASO}

O presente estudo foi aprovado pelo Comitê de Ética em Pesquisa da Associação Educativa do Brasil, SOEBRAS, sob parecer de número 2.755.647, respeitando as diretrizes e critérios estabelecidos pela Resolução 466/12. O presente estudo foi realizado em uma Estratégia de Saúde da Família (ESF) na cidade de Montes Claros - MG, sendo a ESF escolhida por apresentar maior número de gestantes em acompanhamento no município. Tal município é polo educacional e de saúde da região norte de Minas Gerais, situa-se a $16^{\circ} 44^{\prime} 06^{\prime \prime}$ de latitude sul e $43^{\circ} 51^{\prime} 43^{\prime \prime}$ de longitude oeste e sua população é de aproximadamente 402.027 habitantes $^{11}$.

A gestante foi escolhida por ser a única nessa ESF que apresentou a DMG e ainda está com o quadro de gestação gemelar. Para participar do estudo a gestante assinou o Termo de Consentimento Livre e Esclarecido (TCLE) como aceite.

DOI: 10.33947/1982-3282-v15n1-2-4366 
A paciente feminina, M.A.C.S., 33 anos, depiladora, $79,0 \mathrm{~kg} ; 1,65$ metros de altura; casada, ensino médio completo, sendo a gestação gemelar, sua segunda gestação. Sendo essa revelada no primeiro trimestre de gestação, após a realização de uma ultrassonografia realizada em uma clínica particular. No momento do estudo os fetos estavam com 37 semanas, tendo pesos semelhantes, sendo feto I com 2,740 kg e feto II com 2,781 kg, atestado por ultrassonografia.

Relata que a partir do segundo trimestre de gravidez, começou a sentir cansaço além do normal (fadiga), com fraqueza recorrente e perda de peso involuntária. Com isso, o médico clinico geral da ESF que o acompanhava mensalmente no pré-natal, solicitou a realização de exames laboratoriais para mensuração da glicemia e da hemoglobina glicada (HbA1c), no qual com o resultado a paciente foi diagnóstica com a DMG. Descreve ainda não ser portadora do DM anteriormente e sem histórico na família.

A mesma relata que antes de ser diagnostica com a DMG, não se preocupava com a alimentação e nem com a realização atividades físicas. Porém, após o diagnóstico, passou-se realizar o acompanhamento do pré-natal mensalmente em uma clínica medica particular com o profissional especialista em gestação de alto risco. Além disso, passou a utilizar o glicosímetro para realizar aferição da glicemia capilar uma hora antes e após as refeições, e ainda começou a realizar acompanhamento com nutricionista, onde recebeu orientações quanto a sua alimentação, alterando a sua rotina alimentar, fracionamento das refeições com intervalo de três em três horas com o mínimo de quatro refeições diárias, sendo todas preparadas em seu domicílio, priorizando os grelhados/cozidos, comendo em fast-food somente uma vez por mês, sem a ingestão de líquidos durante as refeições.

Inicialmente para o controle da DMG além da dieta prescrita pelo nutricionista, a gestante começou a praticar atividades físicas, contudo não houve sucesso e foi necessário a insulinoterapia duas vezes ao dia durante a gestação.

Após o diagnóstico da DMG, a paciente descreve que passou a conhecer sobre a doença e os fatores de riscos que a mesma poderia acarretar a sua saúde e a dos bebês.

\section{DISCUSSÃO}

As mutações no metabolismo materno são importantes para compor as demandas do feto. No entanto, as mulheres que engravidam com algum grau de resistência à insulina, como nos casos de sobrepeso/obesidade e síndrome dos ovários policísticos, colaborando a ação de hormônios placentários anti-insulínicos são suscetíveis ao quadro de hiperglicemia de potência variada, determinando o DMG tem efeitos adversos maternos e fetais ${ }^{12}$.

Um fator relevante para a incidência da DMG é a idade da gestante, no qual a faixa de risco é considerada com idade igual ou superior a 25 anos 9 . Estando a participante deste estudo dentre essa faixa de risco. Em outro estudo com gestantes portadoras de DMG 60,9\% estavam na faixa etária entre 31 e 40 anos 13. Entretanto, a ocorrência do DMG é cerca de 3 a $6 \%$ maior em gravidez gemelar quando se comparada a gestação de único feto ${ }^{14}$.

Contudo, outros fatores de risco também devem ser levados em consideração como: histórico de diabetes em familiar de primeiro grau, sobrepeso, hipertensão arterial, antecedentes obstétricos de abortos repetitivos, malformações, morte fetal ou neonatal e doença de macrossomia, polidrâmnio ${ }^{9}$.

Em relação ao grau de escolaridade, estudos descrevem que o baixo grau de instrução pode assim também representar um fator de risco, pois, está relacionada à limitação de informações dificultando a estratégia de tratamento 14,15. Não se aplicando a este estudo que a participante tem um grau médio de instrução e acesso a informações.

Observa-se que os peso dos fetos são semelhantes $(2,382 \mathrm{~kg} \pm 0,408 \mathrm{~g})$ aos encontrados por Guillén-Sacoto 19 , porém estes valores são inferiores a peso de fetos de gestação única $3,206 \mathrm{~kg} \pm 0,381 \mathrm{~kg} 19$. Essa diferença pode ser justificada pois em gestações gemelares associada com DMG ocorrem RCF e baixo peso ao nascer ${ }^{20,21}$.

Ter uma alimentação saudável durante a gravidez é essencial para o crescimento saudável do bebê e para o bem-estar da mãe. Diante disso todas as mulheres, em todas as gestações, deveriam ser aconselhadas acerca do consumo de macronutrientes e micronutrientes mais adequado para conseguirem o melhor resultado possível para a sua gravidez ${ }^{16}$. A dieta nutricional deve abranger seis a sete refeições diárias, sendo três refeições principais, duas a três refeições intermédias e uma refeição antes de deitar. A dieta deve proporcionar calorias e nutrientes suficientes, para as necessidades decorrentes da gravidez ${ }^{17}$. 
Sendo assim, para o controle da DMG a dieta acompanhada com da prática de atividades físicas é a primeira opção, contudo estimam que até $60 \%$ das pacientes com diagnóstico de DMG, ocorrem uma falha nessa opção e faz-se necessário o uso de insulinoterapia, dependendo dos para controle glicêmico ${ }^{22}$. Isso foi evidenciado por esse estudo, na qual paciente relata precisou de fazer a utilização da insulina duas vezes ao dia durante a gestação para o controle da glicose.

O conhecimento sobre a doença é a base para a precaução adequada de novas complicações, assim, há uma necessidade maior de transpassar para as pacientes com clareza e simplicidade sobre a patologia ${ }^{18}$.

Diante desse exposto, é importante que os profissionais da saúde estejam altamente qualificados para desenvolver intervenções diretamente ligadas a esses fatores de ricos, de maneira que possa melhorar a qualidade de vida e bem-estar materno e fetal.

\section{CONCLUSÃO}

Verificou-se com esse estudo que a paciente desenvolveu a DMG possivelmente em proveniência da gestação gemelar. O diagnóstico da DMG mudou o acompanhamento do pré-natal em virtude os riscos que essa patologia pode trazer para o feto e para a mãe, podendo levar até ao óbito. A partir disso, a paciente buscou em conhecer mais sobre a DMG e isso influenciou em uma mudança de seus hábitos alimentares na busca por uma gestação com menos riscos maternos e neonatais e com melhorias na qualidade de vida.

\section{CONFLITOS DE INTERESSE}

Os autores declaram não haver conflitos de interesse.

\section{REFERÊNCIAS}

1.Sociedade Brasileira de Diabetes SBD. Diretrizes da Sociedade Brasileira de Diabetes - 2017-2018. São Paulo: Clannad; 2017.

2.American Diabetes Association ADA. Standards of medical care in diabetes - 2020. Diabetes Care. 2020;43(1):1-2.

3.Garcia LFS, Manna TD, Passone CGB, Oliveira LS. Translation and validation of Pediatric Quality of Life InventoryTM 3.0 Diabetes Module (PedsQLTM 3.0 Diabetes Module) in Brazil-Portuguese language. J. Pediatr. 2018;94(6):680-688.

4.Huri HZ, Huey CC, Mustafa N, Mohamad NF, Kamalden TA. Association of glycemic control with progression of diabetic retinopathy in type 2 diabetes mellitus patients in Malaysia. Braz. Braz. J. Pharm. Sci. 2018;54(2):17484.

5.Brasil. Ministério da Saúde. Diabetes: tipos, causas, sintomas, tratamento e prevenção; 2019.

6.International Diabetes Federation. IDF diabetes atlas. 7th ed. Brussels, Belgium: International Diabetes Federation; 2015.

7.Rubin RR, Peyrot M, Kruger DF, Travis LB. Barriers to insulin injection therapy: patient and health care provider perspectives. Diabetes Educ. 2009;35(6):1014-22.

8.Nogueira M, Otuyama LJ, Rocha PA, Pinto VB. Intervenções farmacêuticas no diabetes mellitus tipo 2: uma revisão sistemática e metanálise de ensaios clínicos randomizados. Einstein. 2020;18:eRW4686.

9.Jacob TA, Soares LR, Santos MR, Santos LR, Santos ER, Torres GC, et al. Diabetes Mellitus Gestacional: Uma DOI: 10.33947/1982-3282-v15n1-2-4366 
Revisão de Literatura. Braz. J. Surg. Clin. 2014;6(2):33-7.

10.Brasil. Ministério da Saúde. Departamento de Informação e Informática do SUS. Sistema de Informações de Saúde. Estatísticas Vitais - Nascidos Vivos; 2015.

11.Xavier JLS, Mendes MMV, Santos TAX, Borges BKA. Conhecimento e utilização de medicamentos genéricos, similares e de referência por pacientes em unidades básicas de saúde de Montes Claros-MG. Rev Uningá; 2019;56(1):197-204.

12.Bolognani CV, Souza SS, Calderon IMP. Diabetes mellitus gestacional: enfoque nos novos critérios diagnósticos. Com. Ciências Saúde. 2011;22(1):93-00.

13.Borges MCV, Souza Júnior JÁ, Ribeiro LAN, Ribeiro MF, Oliveira PC, Reis RMS, et al. Conhecimento das gestantes sobre o diabetes mellitus gestacional em unidade de pré-natal no Sul de Minas Gerais. Arch Heath Invest. 2017;6(8):348-1.

14.Montenegro CAB, Filho, JR. Gravidez Gemelar. In: Montenegro CAB, Filho, JR. Rezende Obstetrícia. 13a Ed. Rio de Janeiro: Guanabara Koogan; 2017.

15.Costa LD, Cura CC, Perondi AR, França VF, Bortoloti DS. Perfil epidemiológico de gestantes de alto risco. Cogitare Enferm. 2016;21(2):01-8.

16.Brito CP, Duarte, R. Recomendação global da "international diabetes federation" sobre gravidez e diabetes. Rev. Por. Diab. 2010;5(2):83-90.

17.Abecasis MP. A gravidez, o aumento de peso e o acompanhamento nutricional: custos e benefícios. [dissertação]. Lisboa: Universidade de Lisboa. Mestrado em Nutrição. Faculdade de Medicina de Lisboa; 2015.

18.Mançu TS, Almeida OSC. Conhecimentos das gestantes diabéticas sobre a diabetes mellitus gestacional e tratamento. Rev enferm UFPE on line. 2016;10(3):1474-2.

19.Guillén-Sacoto MA, Barquiel B, Hillman N, Burgos MA, Herranz L. (2018). Diabetes mellitus gestacional: controle glicêmico durante a gravidez e sua relação com desfechos neonatais em gestações gemelares e gestações únicas. Endocr, Diab. Nutr. 2018;65(6):319-327.

20.Gezer A, Rashidova M, Güralp O, Ocer F. Perinatal mortality and morbidity in twin pregnancies: The relation between chorionicity and gestational age at birth. Arch Gynecol Obstet. 2012;285:353-0.

21.Sibai BM, Hauth J, Caritis S, Lindheimer MD, MacPherson C, Klebanoff M, et al. Hypertensive disorders in twin versus singleton gestations, National Institute of Child Health and Human Development Network of Maternal-Fetal Medicine Units. Am J Obstet Gynecol. 2000;182:938-2.

22.Silva JC, Pacheco CP, Bizatto J, Bertini AM. Hipoglicemiantes orais na gestação: metformina versus glibenclamida. Rev. Femina. 2009;37(12):667-670. 2017;21(3):147-153. 